\title{
CLUSTERS VAREJISTAS: CARACTERÍSTICAS RESPONSÁVEIS PELA ATRAÇÃO E AFASTAMENTO DE CONSUMIDORES
}

\section{RETAIL CLUSTERS: FEATURES RESPONSIBLES FOR ATTRACTION AND REJECTION OF CONSUMERS}

João Paulo Lara Siqueira Mestrado Profissional em Administração Gestão do Esporte da Universidade Nove de Julho UNINOVE siqueira.jp@uol.com.br

Renato Telles Programa de Mestrado Acadêmico em Administração - Universidade Paulista - UNIP rtelles@unip.br

Marcus Eduardo Rocca Universidade Paulista - UNIP marcus_rocca@ hotmail.com

Marcos Antônio Gaspar Núcleo de Estudos Avançados em Informática Aplicada - Universidade Nove de Julho UNINOVE marcos_gaspar@hotmail.com

Submissão: 01/12/2013

Aprovação: 05/03/2015 


\section{RESUMO}

O objetivo da pesquisa foi identificar e hierarquizar características de clusters varejistas associadas à atração ou ao afastamento de consumidores. Foi adotada a definição operacional que "um cluster é uma aglomeração considerável de firmas em uma área espacialmente delimitada na qual há um perfil diferenciado de especialização e existem negócios e especialização entre empresas de forma substancial" (ALTENBURG; MEYER-STAMER, 1999). O referencial teórico para atração de clientes aos clusters comerciais baseou-se em cinco trabalhos de Teller publicados entre 2008 e 2012 e também em outros pesquisadores. Primeiramente foi realizada uma investigação exploratória, para a identificação de variáveis potencialmente relacionadas ao fenômeno em questão, seguida por outra etapa, de caráter descritivo, com a aplicação de um questionário a uma amostra não probabilística de 137 consumidores da cidade de São Paulo. As principais conclusões são que, ao analisar a possibilidade de comprar em clusters varejistas, os consumidores são atraídos por características ligadas à conveniência do ato de comprar e a sua experiência anterior em clusters, ao passo que a falta dessa conveniência e a percepção de que os produtos dos clusters não são finos os afastam dos clusters.

Palavras-chave: Clusters. Varejo. Atratividade. Localização. 


\begin{abstract}
This research aimed to identify and rank the features of retail clusters related to their attraction or rejection by consumers. The operational definition of cluster used was that "clusters are considerable agglomerations of firms in a particularly delimited spatial area where there is a specific specialization profile and there are business and specialization among enterprises in a substantial way" (ALTENBURG; MEYER-STAMER, 1999). The theoretical framework on the patronage attraction was based on five articles published by Teller between 2008 and 2012 and also on other researchers. The study started with an exploratory investigation to identify variables that could be potentially related to the phenomena of interest, followed by a descriptive phase that consisted in the interview of 137 consumers in the city of São Paulo, chosen not randomly. The main conclusions are that when consumers are deciding whether to buy - or not - in retail clusters, they are attracted by features related to the convenience of shopping and their previous experience on clusters. On the other hand, the lack of convenience and the perception that the goods sold in clusters are not sophisticated stimulate patrons to move away from clusters.
\end{abstract}

Keywords: Clusters. Retail. Attraction. Location. 


\section{Introdução}

A literatura de varejo menciona o fato de que certos tipos de lojas podem se beneficiar de uma localização próxima de seus concorrentes. São considerados seminais nessa área os trabalhos desenvolvidos por Hotelling (1929) e Nelson (1958). Há algum tempo também são conhecidas aglomerações geográficas de lojas, denominadas clusters varejistas (ZACCARELLI, 2004; BERMAN; EVANS, 2001; INGENE, 1984). Essas concentrações varejistas podem ser encontradas em diversos locais, inclusive nas grandes cidades do Brasil. Na cidade de São Paulo, são exemplos dessa configuração de oferta a Rua Santa Ifigênia, com suas lojas de produtos eletrônicos, a Rua São Caetano, com o varejo de vestidos de noiva (SZAFIR-GOLDSTEIN; TOLEDO, 2004) e os bares da Vila Madalena (TELLES et al., 2011).

A área de estratégia denomina como clusters as concentrações geográficas de empresas especializadas e reconhece sua elevada competitividade. Diversos pesquisadores buscaram explicar a fonte dessa vantagem (PORTER, 1998; SCHMITZ, 1992; ALTENBURG; MEYER-STAMER, 1999; ZACCARELLI et al., 2008, ARIKAN, 2009; BOARI et al., 2003). Assim, ainda que de forma não totalmente consolidada e sem se referir especificamente ao segmento do varejo, há estudos sobre as razões que, do ponto de vista do interesse das empresas, podem motivar a operação de um negócio em um cluster. Com relação à escolha do local para instalar uma operação varejista, deve-se considerar também que se trata de uma decisão estratégica (LITZ; RAJAGURU, 2008; LEVY; WEITZ, 2000; PARENTE, 2000), sendo "crítica porque irá determinar, em grande medida, decisões operacionais relativas a produtos, preços e promoções" (BEISEL, 1993, p. 104) a serem tomadas posteriormente.

A questão da localização comercial mereceu atenção de diversos autores, que elaboraram modelos para a escolha de um local para fazer compras, como é o caso da Lei de Reilly (MASON; BURNS, 1998), da teoria do lugar central de Christaller (LEVY; WEITZ, 2000) e da Lei de Huff (BERMAN; EVANS, 2001). Esses modelos não foram criados especificamente para o caso dos clusters de lojas, mas preocuparam-se em incorporar o interesse dos consumidores, quer seja considerando a distância que eles terão que percorrer para fazer compras ou a variedade de produtos ofertada.

$\mathrm{Na}$ área de varejo, os modelos clássicos para a escolha da localização comercial foram desenvolvidos por pesquisadores inseridos na realidade europeia ou norte-americana e, embora alguns deles levem em consideração agrupamentos de lojas, não foram concebidos 
especificamente para o caso dos clusters. Por outro lado, a pesquisa sobre clusters varejistas apresenta uma produção relativamente baixa (TELLER; REUTTERER, 2008). Um estudo bibliométrico nos periódicos nacionais de Administração, Economia e Engenharia, classificados de A1 a B3, entre os anos de 2000 e 2011, identificou 80 artigos sobre clusters e APLs, mas nenhum deles abordando os clusters comerciais (MASCENA; FIGUEIREDO; BOAVENTURA, 2013).

Pode-se admitir, a partir dessas informações, a ausência de estudos, modelos, explicações e dados sobre como os consumidores posicionam-se quando um cluster varejista é uma alternativa para suas compras. Nesse sentido, o presente estudo adotou como problema de pesquisa a inexistência efetiva de construtos ou esforços de modelagem envolvendo percepção, atitude e/ou conduta de consumidores finais em relação a visita, sondagem e compra em clusters comerciais. Com o propósito de focalização dos esforços de investigação, definiram-se as seguintes questões de pesquisa:

1. Quais características dos clusters varejistas atraem os consumidores e qual a sua importância relativa nesse processo?

2. Quais características dos clusters varejistas afastam os consumidores e qual a sua importância relativa nesse processo?

Em se reconhecendo a ausência efetiva de informações de caráter prático ou teórico sobre o que os consumidores consideram atraente nos clusters varejistas, configura-se como oportuna a investigação sobre a capacidade de os clusters varejistas atraírem clientes. Nesse sentido, o objetivo geral desta pesquisa constitui-se na identificação de características de clusters varejistas associadas à atração ou ao afastamento de consumidores. Como objetivos específicos, foram admitidos:

1. Levantamento e hierarquização por importância de variáveis de clusters varejistas ligadas à atração de consumidores finais; e

2. Levantamento e hierarquização por importância de variáveis de clusters varejistas ligadas ao afastamento de consumidores finais.

\subsection{Justificativa do estudo}

A identificação de características dos clusters comerciais relacionadas à capacidade de atração de clientes é uma contribuição à área de estratégia e estudos organizacionais, que concentra as pesquisas sobre clusters de negócios em administração de empresas. Como, em 
geral, "a quantidade de pesquisas empíricas focalizando o comportamento de compra em aglomerações varejistas, mais especificamente em ruas de comércio ou centros de cidade, pode ser considerada como bastante limitada" (TELLER; REUTTERER, 2008), a busca por evidências empíricas relativas à atratividade dos clusters pode ajudar na descrição e compreensão dessas aglomerações, sendo essa uma contribuição aos estudos da área de estratégia e de clusters e redes de negócio.

Além disso, há a contribuição, na área de varejo, nos estudos sobre localização de pontos comerciais. Informações sobre os clusters como alternativa de localização para lojas podem tornar mais completas as teorias e modelos atualmente empregados, notadamente na realidade brasileira, beneficiando pesquisadores e executivos do varejo.

\section{Referencial teórico}

\subsection{Clusters de negócios}

Desde o trabalho pioneiro de Alfred Marshall (1982), que ao final do século XIX chamou de "indústria localizada" (p. 231) as concentrações manufatureiras em certos locais da Inglaterra que produziam alguns poucos artigos difundidos por toda a Europa, essas aglomerações de empresas, atualmente conhecidas como clusters, passaram a receber a atenção de pesquisas, segundo Schmitz e Nadvi (1999), especialmente em "algumas linhas do novo mainstream da economia (KRUGMAN, 1991), da economia aplicada à administração (PORTER, 1990), da ciência regional (SCOTT, 1996) e dos estudos da inovação (BRACZYK; COOKE; HEIDENREICH, 1998)".

Clusters de empresas podem ser entendidos simplesmente como "concentrações setoriais e espaciais de firmas" (SCHMITZ; NADVI, 1999) ou "concentrações geográficas de companhias e instituições interconectadas em um campo particular" (PORTER, 1998). Alguns autores incorporam à definição o fato de esses agrupamentos apresentarem algum tipo de vantagem para seus membros e os definem como "concentrações de firmas que são capazes de produzir sinergia por causa de sua proximidade geográfica e interdependência" (ROSENFELD, 1997) ou "concentrações locais de certas atividades econômicas" que apresentariam uma "eficiência coletiva" (ALTENBURG; MEYER-STAMER, 1999).

Essas considerações corroboram a premissa de que a localização estimula cooperação e competição entre as empresas estabelecidas próximas umas às outras, fornecendo a base 
para a construção da vantagem competitiva do cluster e de suas firmas (BOARI et al., 2003). Essa eficiência coletiva teria origem em externalidades positivas, custos de transação baixos e ações conjuntas de seus participantes (ALTENBURG; MEYER-STAMER, 1999). Kultti (2011) estudou teoricamente os preços de equilíbrio para uma distribuição arbitrária da demanda em mercados com e sem cluster e demostrou que o mercado com cluster oferece lucros maiores do que o mercado sem cluster.

Os clusters também podem ser compreendidos como localidades onde companhias possuem várias formas de interdependência, como organismos em uma biosfera. Os negócios competem entre si por participação de mercado, empregados e recursos, até mesmo mais vigorosamente do que aqueles que estão fora do cluster, o que torna o conjunto uma economia de alto desempenho (PERRY, 2005). Tais vantagens vinculadas aos clusters tornam-nos particularmente interessantes para a área de estratégia, que neles identifica uma natureza sistêmica. Nesse sentido, Zaccarelli et al. (2008) afirmam que o conjunto de empresas de um cluster forma um sistema que pode ser entendido como uma entidade supra empresarial, com comportamento e características próprias, não encontradas nas empresas isoladamente.

Atualmente, os clusters são reconhecidos como elementos importantes das estratégias urbanas e regionais (YANG; HAO; CAI, 2015), estando ligados ao desenvolvimento regional (YANG; HAO; CAI, 2015; ZACARELLI et al., 2008; PORTER, 1998). Martin e Mayer (2008) afirmam que o desenvolvimento econômico de um país depende de sua habilidade em nutrir e desenvolver clusters de indústrias que sejam competitivos. Vale notar que, apesar de clusters poderem ser encontrados em praticamente todo o mundo, um particular caso de cluster, as concentrações espaciais de empresas de pequeno e médio porte, recebeu atenção de pesquisadores, como o caso dos distritos industriais italianos (PIORE; SABEL, 1984; BECATTINI, 1991).

Alguns pesquisadores entendem que ainda há considerável confusão sobre o significado de cluster em relação a outros tipos de aglomeração, não sendo viável uma definição clara e rigorosa para o conceito (MARTIN; SUNLEY, 2003, apud MALMBERG; POWER, 2005). No presente estudo, utilizou-se a definição operacional adotada por Altenburg e Meyer-Stamer (1999), de que "um cluster é uma aglomeração considerável de firmas em uma área espacialmente delimitada na qual há um perfil diferenciado de especialização e existem negócios e especialização entre empresas de forma substancial". 


\subsection{Clusters varejistas}

Em que pese a literatura sobre os clusters abordar com maior frequência as aglomerações de manufaturas, como os casos descritos no livro Vantagem competitiva das nações, de Michael Porter (1990), encontram-se com frequência concentrações geográficas de empresas em outros segmentos de atividade, como os clusters de serviços financeiros em Nova York, de cassinos em Las Vegas, de turismo em Portugal (PORTER, 1998), de serviços médicos em Recife (FERNANDES; LIMA, 2006) e de turismo focado em destinos de estilo de vida saudável no Reino Unido (NOVELLI et al., 2006). No setor varejista também há casos de agrupamentos de empresas, como os centros comerciais não planejados que têm evolução espontânea e situam-se em locais de concentração demográfica elevada e próximos às intersecções da malha viária das cidades (PARENTE, 2000). Tal aglomeração varejista aumenta a atratividade local, tanto no caso dos deslocamentos dos clientes para compras com propósito único, como também para propósito múltiplo (ARENTZE et al., 2005). Nessas áreas, a saturação comercial "oferece aos clientes boa seleção de mercadorias e serviços, enquanto permite que os varejistas tenham bons lucros" (LEVY; WEITZ, 2000, p. 235).

$\mathrm{O}$ fato da proximidade com os concorrentes poder ser mais importante para o sucesso dos negócios no varejo do que a proximidade com os clientes foi mencionado de forma pioneira na literatura por H. Hotelling (1929), que o denominou Princípio da Mínima Diferenciação. O trabalho de Hotelling permanece atual. Russell (2013) relata a utilização de simulações em jogos de empresas para facilitar a estudantes a descoberta de sua lógica, aparentemente contrária à intuição. Posteriormente, R. L. Nelson (1958) introduziu o Princípio da Atração Cumulativa, segundo o qual "certo número de lojas que vendem as mesmas mercadorias venderão mais se estiverem localizadas de forma adjacente ou próxima umas das outras, do que se estiverem dispersas".

A complementaridade que surge da concentração de lojas numa determinada área desenvolve o mercado, ainda que às vezes a concorrência torne-se mais acirrada do que quando ele é dividido (BRANDENBURGUER; NALEBUFF, 1999). Pesquisa desenvolvida por Donaire et al. (2013) evidenciou a importância da cooperação entre os participantes de um cluster varejista para o desenvolvimento de sua atração para os clientes. Nesse sentido, estudo conduzido por Marietto e Sanches (2013) acentua a importância do desenvolvimento da estratégia coletiva do cluster para seu êxito. 
Evidências empíricas da presença dos clusters varejistas em diversos locais vêm sendo apresentadas ao longo do tempo: Proudfoot (1937) relatou clusters de lojas isolados em grandes cidades dos EUA; Rogers (1965) menciona uma pesquisa quantitativa sobre clusters comerciais em Estocolmo, na Suécia; Horton (1968) cita clusters comerciais nos EUA e Biba et al. (2008) apresentam casos de clusters de varejo no Canadá. Um cluster comercial é um centro de compras (ou um conjunto de lojas) em uma região geográfica específica que oferece um resultado único e sistêmico e produz sinergia graças a transações comerciais, formas de comunicação e diálogo, que acabam por beneficiar-se das mesmas oportunidades e por enfrentar os mesmos problemas (BIBA et al., 2008; LEE-ROSS, 2008).

\subsection{Características de clusters varejistas ligadas à atração dos clientes}

Do ponto de vista dos varejistas, as concentrações de lojas criam uma situação de coopetição (competição e cooperação) entre seus membros, pois, apesar de disputarem os mesmos clientes, eles beneficiam-se do volume de consumidores atraídos pelo total de lojas da aglomeração e compartilham uma infraestrutura comum (BRANDENBURGUER; NALEBUFF, 1999). Sob a perspectiva dos consumidores, a aglomeração oferece um valor adicional para a atividade de compra, em relação às lojas isoladas (TELLER; REUTTERER, 2008). Telles et al. (2013, p. 54) indicam que a atratividade dos clusters varejistas repousa na "convergência entre a perspectiva do consumidor final e a perspectiva do varejista". O reconhecimento de que a presença da concorrência pode ser um estímulo à atração de clientes para regiões/mercados pode ser observado na estratégia de localização de novas lojas de algumas redes varejistas. "Alguns restaurantes como o Burguer King procuram locais onde seu maior concorrente - o McDonald's - tenha uma forte presença” (LEVY; WEITZ, 2000, p. 235).

O interesse dos consumidores pelas concentrações de lojas pode ser atribuído a diversas características presentes nessas aglomerações. Pesquisadores de varejo, marketing e estratégia elaboraram relações dessas características, que incluem a "disponibilidade de ampla linha de produtos e preços confiáveis" (ZACCARELLI, 2004, p. 199); a possibilidade de comparar preços e produtos; a acessibilidade (transporte fácil para o local); a existência de estacionamento; o sortimento amplo de produtos, preços bons e eventual existência de varejo complementar, como lugares para comer e lazer (KIM, 2002); o sortimento de lojas (o tenant 
mix); a variedade dos produtos; o valor da mercadoria e o tipo dos vendedores; a atmosfera do local; a sinalização e os equipamentos de infraestrutura (TELLER; ELMS, 2010).

Os resultados de um levantamento no qual 500 consumidores foram entrevistados no local de compra revelaram que o sortimento de lojas e a atmosfera do local são os fatores que influenciam a atração do aglomerado de forma mais significativa (TELLER et al., 2010), mas secundariamente também contribuem para isso as condições de estacionamento, o sortimento de empresas não varejistas, a facilidade para manobrar e a orientação (TELLER; ELMS, 2012).

As características de clusters relatadas na literatura não se repetem em todos os estudos e, dependendo do país, apresentam relevantes especificidades. Investigação realizada nos Emirados Árabes Unidos revelou seis fatores de atração de consumidores às aglomerações de lojas: conforto, possibilidade de entretenimento, diversidade (de lojas), essência do conjunto de lojas (aspecto ligado ao estilo do cluster), conveniência e luxo (ELADLY, 2007). Os clusters comerciais também apresentam características que podem desestimular indivíduos a considerá-los como opção preferencial para compras. Telles et al. (2013) enfatizam aspectos mandatórios à atratividade dos clusters varejistas, tais como as condições de compra e os preços oferecidos pelas lojas atuantes nessas aglomerações comerciais. Outros atributos, ligados a um possível afastamento dos clientes de clusters varejistas, seriam (a) energia gasta para enfrentar vendedores desagradavelmente insistentes, (b) espera excessiva em filas no caixa e (c) problemas de segurança na realização das compras. Outras características, como apelo aos cinco sentidos, sinalização existente e arquitetura do local, podem atrair ou afastar os clientes em função de sua avaliação pelos consumidores ser considerada positiva ou não (KIM, 2002).

A Figura 1 apresenta o entendimento, admitido neste estudo, do processo de avaliação da capacidade de atração e afastamento de clientes de um cluster comercial, em função de suas características. Sob essa perspectiva, a decisão de escolha por um determinado cluster como opção de compras resulta da percepção do consumidor sobre as características desse agrupamento. 
Figura 1 - Avaliação da capacidade de atração e afastamento de clientes de clusters comerciais

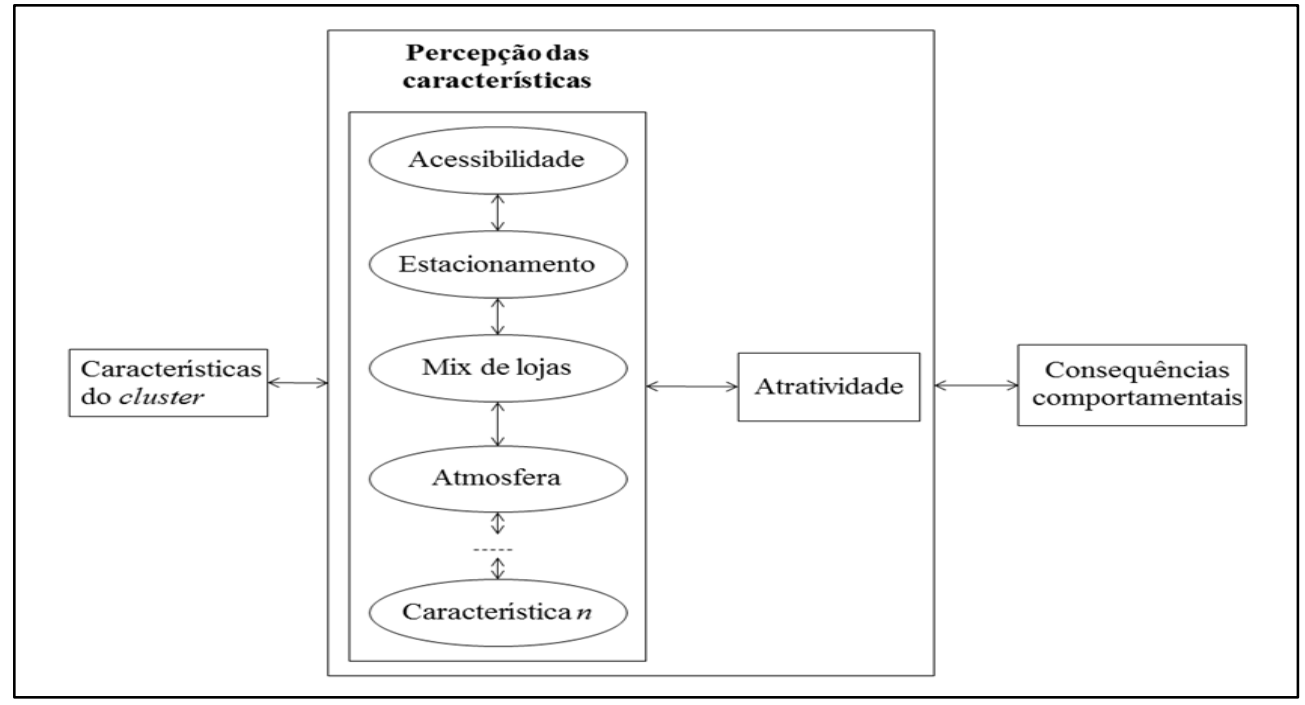

Fonte: Adaptado pelos autores Teller e Reutterer (2008).

Deve-se reconhecer que o construto apresentado refere-se ao exame das características dos clusters varejistas sob uma percepção individual, sendo a capacidade geral de atração de clientes de um cluster o agregado das avaliações individuais dos seus possíveis clientes.

\section{Método empregado}

O percurso metodológico adotado no presente estudo orientou-se para o avanço da compreensão de características potencialmente associadas à atração ou ao afastamento de clientes nos clusters varejistas. A partir dessa perspectiva, tornou-se necessária a constituição de um inventário de variáveis possivelmente relacionadas a essas condutas, o desenvolvimento de um instrumento de coleta focalizado na captura dessas variáveis, o delineamento da população e amostra, e o reconhecimento das ressalvas presentes no trabalho. A pesquisa conduzida pode ser entendida como composta por duas etapas estanques: (1) uma primeira, de natureza exploratória, orientada à identificação de variáveis potencialmente relacionadas à atração e/ou afastamento de clusters varejistas em relação aos consumidores, e (2) uma segunda, de caráter mais descritivo, quando se investigou o comportamento das variáveis, arroladas na fase inicial, para a amostra selecionada da população de interesse da pesquisa. 


\subsection{Etapa exploratória da pesquisa}

$\mathrm{Na}$ etapa exploratória, a investigação foi eminentemente qualitativa, desenvolvida a partir de entrevistas semiestruturadas com dois pesquisadores da área de varejo e dois pesquisadores da área de estratégia que se dedicam ao tema clusters de negócios. A abordagem qualitativa é especialmente recomendada na investigação exploratória. Terence e Escrivão (2006) afirmam que essa prática viabiliza e impulsiona a construção de compreensões inovadoras sobre a diversidade e a profundidade dos fenômenos sociais. A tipificação como qualitativa nesse momento da pesquisa, conforme Gordon e Langmaid (1988), é compatível com a utilização de pequenas amostras, não demandando estatuto de representatividade. Em relação a essa conduta, Churchill (1979) indica, como opções de arrolamento de variáveis, a abordagem do incidente crítico ou do grupo focal, como conduzido neste trabalho. Os pesquisadores propuseram relações de características de clusters varejistas, que poderiam contribuir para atração ou afastamento de clientes. Os resultados dessa etapa encontram-se consolidados nos Quadros 1 e 2, sendo que no primeiro deles encontram-se características que podem ser responsáveis tanto por atrair quanto por afastar clientes, dependendo de suas intensidades. O Quadro 2 apresenta características que afastam clientes.

Quadro 1 - Características dos clusters comerciais que podem atrair ou afastar os clientes

\begin{tabular}{|l|c|}
\hline \multicolumn{1}{|c|}{ ATRIBUTOS } & VARIÁVEL \\
\hline Existência de lojas de redes varejistas conhecidas & LOJCON \\
\hline Rapidez com que as compras são feitas & COMRAP \\
\hline Os preços são confiáveis & PREBXO \\
\hline A variedade de produtos é ampla & VARGDE \\
\hline Os vendedores conhecem os produtos & CONVEN \\
\hline Facilidade para encontrar o que se procura & ENCFAC \\
\hline Facilidade para se informar sobre os produtos & INFFAC \\
\hline Facilidade do acesso ao local (de casa ou do trabalho) & LOCBOA \\
\hline Facilidade para obter transporte para o local & ACEFAC \\
\hline Facilidade para achar produtos que completam compra principal & PRCFAC \\
\hline
\end{tabular}

Fonte: Autores. 
Quadro 2 - Características dos clusters comerciais que afastam os clientes

\begin{tabular}{|l|c|}
\hline \multicolumn{1}{|c|}{ ATRIBUTOS } & VARIÁVEL \\
\hline Há movimento em excesso no local & MOVDEM \\
\hline Os produtos vendidos não são finos & PRONFN \\
\hline Os produtos são todos do mesmo fornecedor (fabricante) & MMFABR \\
\hline Os vendedores enganam os clientes & VENDCH \\
\hline O local não é seguro & SEMSEG \\
\hline É ruim ir sem carro e voltar carregando compras & RUIMSC \\
\hline Facilidade para estacionar o carro & ESTFAC \\
\hline
\end{tabular}

Fonte: Autores.

Os resultados da primeira etapa do estudo foram confrontados com o conteúdo presente na revisão da literatura e, com base nessas informações, foi elaborado o instrumento de coleta de dados, com predominância de questões fechadas, aperfeiçoado após pré-teste com 13 entrevistados.

\subsection{Instrumento de pesquisa}

Com o propósito de verificar se o entrevistado era cliente de clusters comerciais, solicitou-se aos respondentes indicar o local preferido para realizar compras de oito produtos: lustres, vestidos de noiva, madeiras (e material para marcenaria), ferramentas, automóveis usados, instrumentos musicais, eletroeletrônicos e informática. Caso o local indicado para mais da metade dos produtos fosse um cluster de lojas, arbitrou-se admitir que o respondente fosse um cliente de clusters comerciais. A avaliação dos entrevistados das características de clusters comerciais que podem atrai-los ou afastá-los, assim como sua avaliação sobre suas importâncias, foi coligida por meio da atribuição de pontos em uma escala contínua com rótulos bipolares nas extremidades, relativos a afirmações envolvendo tais características. $\mathrm{O}$ Quadro 3 apresenta a estrutura utilizada. 
Quadro 3 - Escalas de características que atraem e afastam clientes dos clusters varejistas

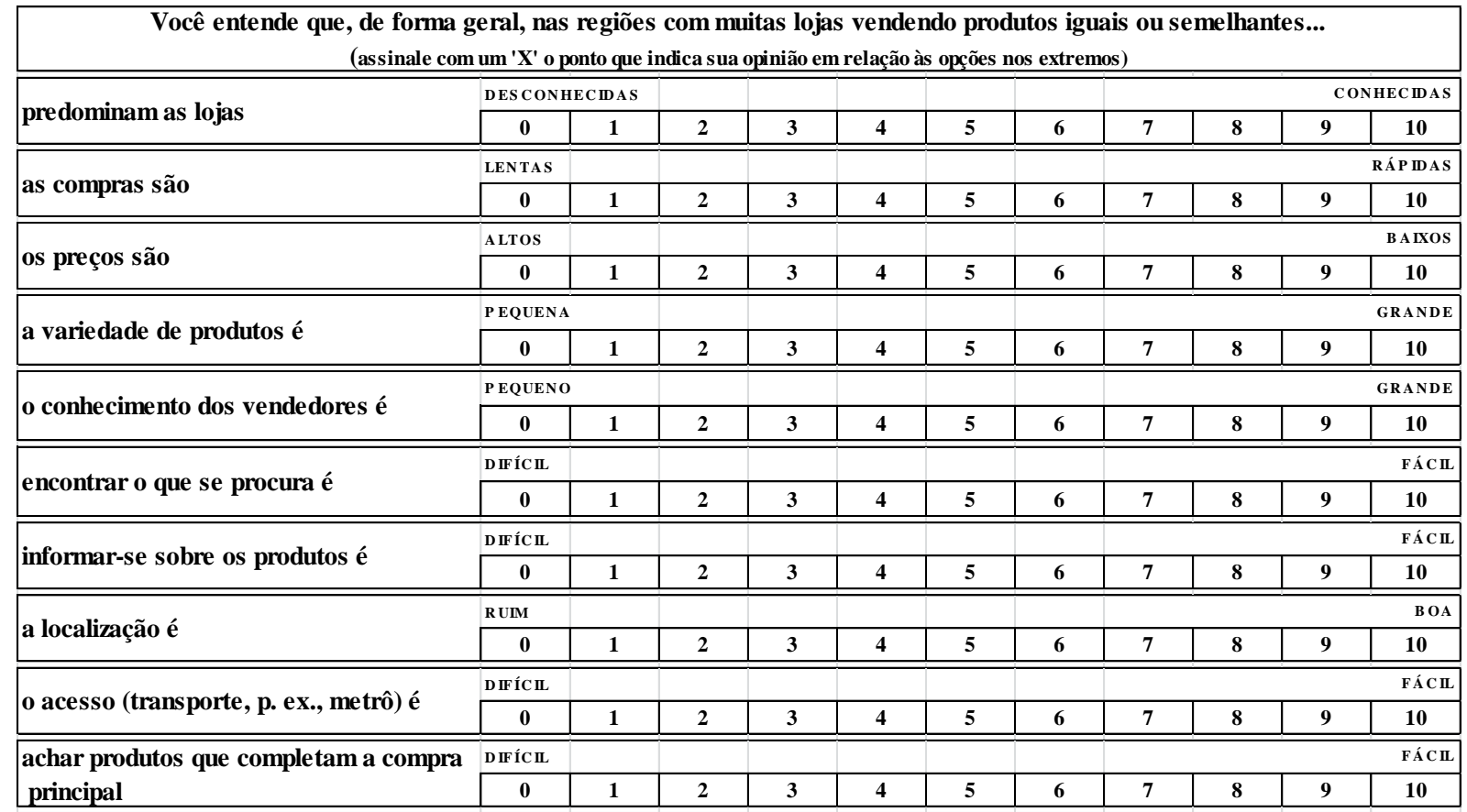

Fonte: Autores.

Escalas em que entrevistados posicionam-se em relação a rótulos bipolares são conhecidas na literatura de pesquisa de marketing como diferencial semântico (MALHOTRA, 2006; MCDANIEL; GATES, 2003). Tendo em vista a concordância entre revisão da literatura e informações dos especialistas, apontando a existência de características associadas ao afastamento de indivíduos das aglomerações de lojas, o instrumento de pesquisa incorporou questões que solicitavam aos entrevistados a informação se, em sua opinião, em geral, os clusters comerciais possuem tais características, conforme apresentado no Quadro 4.

Quadro 4-Questões sobre características que afastam consumidores dos clusters varejistas

\begin{tabular}{|l|l|l|}
\hline 1. Há movimento demais (muita gente comprando) & ( )Sim & ( ) Não \\
\hline 2. As compras não são rápidas & ( )Sim & ( ) Não \\
\hline 3. Os produtos à venda não são finos & ( )Sim & ( ) Não \\
\hline 4. Os produtos são todos do mesmo fabricante (ou fornecedor) & ( )Sim & ( ) Não \\
\hline 5. Os vendedores incomodam os clientes & ( )Sim & ( ) Não \\
\hline 6. É difícil encontrar o que se procura & ( )Sim & ( ) Não \\
\hline 7. Os preços estão na média dos outros lugares & ( )Sim & ( ) Não \\
\hline 8. Não há segurança (há assaltos, furtos etc.) & ( )Sim & ( ) Não \\
\hline 9. É difícil estacionar o carro & ( )Sim & ( ) Não \\
\hline 10. É ruim ir sem carro e depois voltar carregando muitas compras & ( )Sim & ( ) Não \\
\hline
\end{tabular}

Fonte: Autores. 
A síntese da arquitetura do instrumento de coleta encontra-se representada esquematicamente no Quadro 5.

Quadro 5 - Síntese da arquitetura do instrumento de coleta utilizado (identificando variáveis)

\begin{tabular}{|c|c|c|c|}
\hline $\mathbf{N}^{\mathbf{o}}$ & VARIÁVEL & ESCALA & NOME \\
\hline 1 & Sexo & M (1) ou F (2) & GENERO \\
\hline 2 & Já fez compra nos clusters & $\mathrm{S}(1)$ ou $\mathrm{N}(0)$ & COMPRA \\
\hline 3 & Já foi ao cluster e não comprou & S (1) ou N (0) & VISITA \\
\hline 4 & Conhece a maioria dos clusters & S (1) ou N (0) & CONHEC \\
\hline 5 & No cluster predominam as lojas desconhecidas $\mathrm{x}$ conhecidas & $\mathrm{S}(1)$ ou N $(0)$ & LOJCON \\
\hline 6 & No cluster as compras são lentas x rápidas & S (1) ou N (0) & COMRAP \\
\hline 7 & No cluster os preços são altos x baixos & $\mathrm{S}(1)$ ou $\mathrm{N}(0)$ & PREBXO \\
\hline 8 & No cluster a variedade de produtos é pequena $\mathrm{x}$ grande & S (1) ou N (0) & VARGDE \\
\hline 9 & $\begin{array}{l}\text { No cluster o conhecimento dos vendedores sobre os produtos é pequeno } \mathrm{x} \\
\text { arande }\end{array}$ & $\mathrm{S}(1)$ ou $N(0)$ & CONVEN \\
\hline 10 & No cluster encontrar o que se procura é difícil x fácil & 0 -------- 10 & ENCFAC \\
\hline 11 & No cluster se informar sobre os produtos difícil $\mathrm{x}$ fácil & 0 -------- 10 & INFFAC \\
\hline 12 & No cluster a localização é ruim x boa & 0 ------- 10 & LOCBOA \\
\hline 13 & No cluster o acesso (meios de transporte, por ex., metrô) é difícil x fácil & 0 --------- 10 & ACEFAC \\
\hline 14 & No cluster achar produtos que completam a compra principal é difícil x fácil & 0 --------- 10 & PRCFAC \\
\hline 15 & No cluster há movimento demais (muita gente comprando) & $\mathrm{S}(1)$ ou $\mathrm{N}(0)$ & MOVDEM \\
\hline 16 & No cluster as compras não são rápidas & S (1) ou N (0) & COMDEM \\
\hline 17 & No cluster os produtos à venda não são finos & $\mathrm{S}(1)$ ou $\mathrm{N}(0)$ & PRONFN \\
\hline 18 & No cluster os produtos são todos do mesmo fabricante (ou fornecedor) & $\mathrm{S}(1)$ ou $\mathrm{N}(0)$ & MMFABR \\
\hline 19 & No cluster os vendedores enrolam os clientes & S (1) ou N (0) & VENDCH \\
\hline 20 & No cluster é difícil achar o se que procura & $\mathrm{S}(1)$ ou $\mathrm{N}(0)$ & ENCDIF \\
\hline 21 & No cluster os preços estão na média dos outros lugares & $\mathrm{S}(1)$ ou $\mathrm{N}(0)$ & PREMED \\
\hline 22 & No cluster não há segurança (há assaltos, furtos etc.) & $\mathrm{S}(1)$ ou N (0) & SEMSEG \\
\hline 23 & No cluster é difícil es tacionar o carro & $\mathrm{S}(1)$ ou $\mathrm{N}(0)$ & ESTFAC \\
\hline 24 & É ruim ir ao cluster sem carro e depois voltar carregando muitas compras & S (1) ou N (0) & RUIMSC \\
\hline
\end{tabular}

Fonte: Autores.

A estrutura do questionário foi desenvolvida no sentido de capturar (1) variáveis categóricas e numéricas de discriminação geral do respondente (basicamente gênero e sua proximidade com a experiência de pesquisa e compra em clusters varejistas), (2) variáveis ordinais e numéricas das posições em relação às dez variáveis desenvolvidas e potencialmente vinculadas à atração e ao afastamento dos clientes em relação a clusters de lojas, e (3) variáveis categóricas e numéricas para crenças sobre as dez variáveis passíveis de associação com o afastamento de consumidores de clusters de varejo. 


\subsection{Etapa descritiva da pesquisa}

Na segunda fase da investigação, a pesquisa deve ser entendida como descritiva, visto que se propõe a "descrever as características de grupos relevantes, como consumidores, vendedores, organizações ou áreas de mercado" (MALHOTRA, 2006, p. 101), obtendo um instantâneo do ambiente de mercado (AAKER et al., 2004). O estudo retrata uma situação em certo momento, constituindo, portanto, um corte transversal.

Os dados foram coletados no segundo semestre de 2012. A população de interesse do estudo é composta por pessoas residentes em grandes cidades, aptas a escolher local de suas compras. Considerando esse contexto, optou-se pela aplicação do questionário a maiores de 18 anos, de ambos os sexos, residentes na cidade de São Paulo. A amostragem, em função da inviabilidade de demarcação ou seleção aleatória de elementos da população, foi realizada por disponibilidade e não é probabilística. Foram realizadas 137 entrevistas com pessoas selecionadas ao acaso em locais públicos de elevado movimento urbano. Apenas a título de comparação, vale lembrar que uma pesquisa com amostragem probabilística, cuja pergunta mais importante é dicotômica, com uma amostra de 137 elementos de uma população infinita - a um nível de confiança de $90 \%$ - é compatível com um erro máximo de 5\% no processo de inferência. O Quadro 6 apresenta os 48 bairros onde foram coletados os dados, agrupados por região da cidade.

Quadro 6 - Regiões e bairros das entrevistas

\begin{tabular}{|c|l|}
\hline REGIÃO DA CIDADE & \multicolumn{1}{c|}{ BAIRROS } \\
\hline Centro & Bela Vista, Bom Retiro, Liberdade. \\
\hline Norte & $\begin{array}{l}\text { Cachoeirinha, Freguesia do Ó, Imirim, Horto Florestal, Lauzane Paulista, } \\
\text { Limão, Perus, Pirituba, Santana, Vila Maria, Vila Nova Cachoeirinha. }\end{array}$ \\
\hline Sul & $\begin{array}{l}\text { Aclimação, Brooklin, Butantã, Campo Belo, Campo Limpo, Chácara Sto. } \\
\text { Antonio, Cidade Ademar, Cursino, Interlagos, Ipiranga, Jabaquara, Jardins, Jd. } \\
\text { Marajoara, Morumbi, Paraíso, Pedreira, Pinheiros, Planalto Paulista, Sacomão, } \\
\text { Saúde, Vila Clementino, Vila das Mercês, Vila Mascote, Vila Monumento. }\end{array}$ \\
\hline Leste & Guainazes, Mooca, Penha, São Mateus, Sapopemba, Tatuapé. \\
\hline Oeste & Jaraguá, Lapa, Sta. Cecília, Vila dos Remédios. \\
\hline
\end{tabular}

Fonte: Autores.

As limitações metodológicas desta pesquisa estão associadas principalmente ao processo de coleta de dados suportado por instrumentos de pesquisa estruturados com auxílio de entrevistador. Como a maioria das perguntas é fechada, limita-se a viabilidade de captação 
de detalhes e aprofundamentos que investigações qualitativas permitiriam. Em se tratando de um corte transversal, as informações obtidas referem-se ao momento de execução da pesquisa, limitando os resultados a esse contexto. A amostragem adotada não constitui efetivamente processo probabilístico de coleta de dados, a rigor, não oferecendo possibilidade de generalizações. Entretanto, as informações obtidas pela qualificação da amostra oferecem uma plataforma adicional de referência para estudos futuros.

\section{Análise dos resultados}

A apresentação dos dados coligidos e a análise dos resultados foram organizadas em três seções: (1) Confiabilidade e validade dos construtos, em que se discute a qualidade do instrumento de pesquisa, a confiabilidade e a validade dos construtos; (2) Estatísticas descritivas, com a apresentação dos resultados sobre as posições dos respondentes, enquanto população amostral, para cada uma das variáveis apresentadas; e (3) Análise discriminante.

\subsection{Confiabilidade e validade dos construtos}

Numa primeira avaliação sobre a qualidade do instrumento, utilizaram-se os resultados do pré-teste para se realizar a validação de conteúdo (ou validação facial), conduzida por especialistas no conteúdo das métricas e na representatividade para mensuração (KINNEAR; TAYLOR, 1996; NUNNALLY; BERNSTEIN, 1994; MALHOTRA, 2006), compostos por três doutores em administração. O resultado dessa análise inicial não indicou a presença de sinais de comprometimento do instrumento, conduzindo a análises focalizadas em indicadores específicos sobre a confiabilidade e validade dos construtos.

Uma das dimensões de avaliação crítica de um instrumento construído a partir de uma iniciativa exploratória de identificação de atributos ou variáveis relevantes em um contexto é análise de sua confiabilidade. Por confiabilidade entende-se a precisão com que um conceito (ou construto) é mensurado por um artefato métrico e em que medida esse construto é capaz de reproduzir os mesmos resultados. Um dos principais indicadores ou parâmetros utilizados no exame da confiabilidade é valor do Alfa de Cronbach (COOPER; SCHINDLER, 2003; VAN DER VELDE; JANSEN; ANDERSON, 2004) (Tabela 1). 
Tabela 1 - Confiabilidade dos construtos

\begin{tabular}{|c|c|c|c|c|}
\hline VARIÁVEL & CONSTRUTO & $\begin{array}{c}\text { Alfa } \\
\text { Cronbach }\end{array}$ & $\begin{array}{l}\text { Alfa Cronbach } \\
\text { Padronizado }\end{array}$ & $\begin{array}{l}\text { Número de } \\
\text { itens }\end{array}$ \\
\hline LOJCON & Lojas conhecidas no cluster & 0,807 & 0,808 & 20 \\
\hline COMRAP & Compra rápida no cluster & 0,804 & 0,807 & 20 \\
\hline PREBXO & Preço baixo no cluster & 0,772 & 0,778 & 20 \\
\hline VARGDE & Variedade grande no cluster & 0,741 & 0,749 & 20 \\
\hline CONVEN & $\begin{array}{c}\text { Conhecimento dos vendedores no } \\
\text { cluster }\end{array}$ & 0,771 & 0,779 & 20 \\
\hline ENCFAC & Encontrar produtos é fácil & 0,676 & 0,690 & 20 \\
\hline INFFAC & Obter informação é fácil & 0,743 & 0,755 & 20 \\
\hline LOCBOA & Localização do cluster é boa & 0,757 & 0,773 & 20 \\
\hline ACEFAC & Acesso ao cluster é fácil & 0,793 & 0,812 & 20 \\
\hline PRCFAC & Produtos adicionais à compra é fácil & 0,711 & 0,730 & 20 \\
\hline MOVDEM & Movimento demais de compradores & 0,809 & 0,833 & 20 \\
\hline COMDEM & Compras no cluster são demoradas & 0,813 & 0,840 & 20 \\
\hline PRONFN & Produtos no cluster não são finos & 0,815 & 0,844 & 20 \\
\hline MMFABR & Produtos são do mesmo fabricante & 0,811 & 0,842 & 20 \\
\hline VENDCH & Vendedores enganam os clientes & 0,819 & 0,852 & 20 \\
\hline ENCDIF & É difícil se achar o que se procura & 0,827 & 0,863 & 20 \\
\hline PREMED & Preços estão na média do mercado & 0,820 & 0,835 & 20 \\
\hline SEMSEG & Não há segurança nos clusters & 0,814 & 0,824 & 20 \\
\hline ESTFAC & Estacionamento é fácil nos clusters & 0,810 & 0,828 & 20 \\
\hline RUIMSC & Ruim ir sem carro (carga de compras) & 0,814 & 0,823 & 20 \\
\hline
\end{tabular}

Fonte: Autores.

Hair, Black, Barry e Anderson (2009, p. 118) sugerem que o valor do Alfa de Cronbach seja igual ou superior a 0,7 para estudos em geral e 0,6 para estudos exploratórios como padrões analíticos de avaliação da confiabilidade. Verifica-se, pelos valores expressos na Tabela 1, que mostra os Alfas de Cronbach para cada uma das métricas (construtos) utilizadas no instrumento de coleta, que esses construtos, quando considerados os limites recomendados por Hair et al. (1998), podem ser considerados confiáveis.

Van der Velde, Jansen e Anderson (2004, p. 54) afirmam que a confiabilidade (ou seja, como se processa a medida) é condicionante para a validade (ou seja, se está se medindo o que se deseja medir); assim, um instrumento confiável não necessariamente é capaz de medir o que se propõe a mensurar. Lançou-se mão dos testes de Kayser-Meyer-Olkin de adequação da amostra (KMO) e de esfericidade de Bartlett com o objetivo de aquilatar-se a validade dos construtos utilizados no instrumento de pesquisa. A Figura 1 de forma esquemática remete à condição de validade, em função dos valores apresentados pelos dois indicadores usados. 
Figura 1 - Resultado de testes de KMO e Bartlett (saída do software IBM SPSS v.21.0)

Medida Kaiser-Meyer-Olkin de adequação de amostragem.

Teste de esfericidade de Bartlett

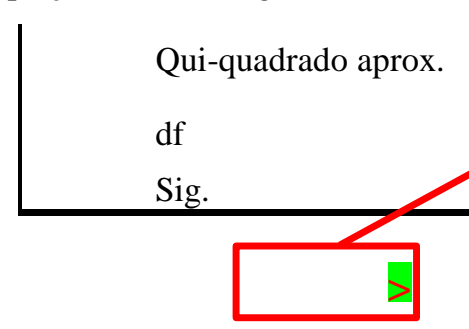

Fonte: Autores.

\subsection{Estatísticas descritivas}

As Tabelas 2 e 3 apresentam, respectivamente, estatísticas descritivas relativas aos fatores que podem atrair ou afastar os clientes dos clusters. As características em que as opiniões aproximam-se mais de um dos extremos da escala são: (a) a variedade dos produtos, que tende a ser vista como 'grande' (média 8,3); (b) o fato de que encontrar o que se procura aproxima-se de ser visto como 'fácil' (média 7,5); (c) o acesso à aglomeração (por meios de transporte) próximo de ser considerado 'fácil' (média 7,1); e (d) a possibilidade de encontrar produtos que complementam a compra principal tende a ser 'fácil' (média 7,1).

Tabela 2 - Médias e desvios-padrão das características que podem atrair ou afastar os clientes

\begin{tabular}{|c|c|c|c|c|}
\hline \multicolumn{5}{|c|}{ Você entende que, de forma geral, nas regiões com muitas lojas vendendo produtos iguais ou semelhantes: } \\
\hline \multirow[b]{2}{*}{ Predominam as lojas } & \multicolumn{2}{|c|}{$\begin{array}{c}\text { Diferenciais Semânticos (com } \\
\text { posições de zero a } 10 \text { para as } \\
\text { opiniões) }\end{array}$} & \multirow{2}{*}{$\begin{array}{c}\text { Média } \\
5,6\end{array}$} & \multirow{2}{*}{$\begin{array}{c}\begin{array}{r}\text { Desvio- } \\
\text { Padrão }\end{array} \\
2,4\end{array}$} \\
\hline & Desconhecidas & Conhecidas & & \\
\hline As compras são & Lentas & Rápidas & 6,0 & 2,0 \\
\hline Os preços são & Altos & Baixos & 6,5 & 1,7 \\
\hline A variedade de produtos é & Pequena & Grande & 8,3 & 1,5 \\
\hline O conhecimento dos vendedores sobre os produtos é & Pequeno & Grande & 6,7 & 1,8 \\
\hline Encontrar o que se procura é & Difícil & Fácil & 7,5 & 1,8 \\
\hline Informar-se sobre os produtos é & Difícil & Fácil & 6,8 & 1,6 \\
\hline A localização é & Ruim & Boa & 6,6 & 2,0 \\
\hline O acesso (meios de transporte, por ex., Metrô) é & Difícil & Fácil & 7,1 & 2,3 \\
\hline Achar produtos que complementam a compra principal é & Difícil & Fácil & 7,1 & 1,7 \\
\hline
\end{tabular}

Fonte: Autores.

Os desvios-padrão das quatro características destacadas são respectivamente 1,5, 1,8, 2,3 e 1,7, estando entre os menores valores para esse parâmetro de todas as características, aspecto que sugere convergência importante das opiniões. 
Tabela 3 - Percentuais das características que podem afastar os clientes

\begin{tabular}{lll}
\hline AFIRMAÇÃO PROPOSTA & SIM & NÃO \\
\hline 1. Há movimento demais (muita gente comprando) & $1,5 \%$ & $98,5 \%$ \\
\hline 2. As compras não são rápidas & $59,1 \%$ & $40,9 \%$ \\
\hline 3. Os produtos à venda não são finos & $57,7 \%$ & $42,3 \%$ \\
\hline 4. Os produtos são todos do mesmo fabricante (ou fornecedor) & $89,8 \%$ & $10,2 \%$ \\
\hline 5. Os vendedores incomodam os clientes & $37,2 \%$ & $62,8 \%$ \\
\hline 6. É difícil encontrar o que se procura & $81,8 \%$ & $18,2 \%$ \\
\hline 7. Os preços estão na média dos outros lugares & $59,1 \%$ & $40,9 \%$ \\
\hline 8. Não há segurança (há assaltos, furtos etc.) & $29,9 \%$ & $70,1 \%$ \\
\hline 9. É difícil estacionar o carro & $2,2 \%$ & $97,8 \%$ \\
\hline 10. É ruim ir sem carro e depois voltar carregando muitas compras & $5,8 \%$ & $94,2 \%$ \\
\hline
\end{tabular}

Fonte: Autores.

A Tabela 3 remete à compreensão de que os entrevistados não consideram a existência de movimento em excesso nos clusters comerciais, em geral, uma vez que 98,5\% responderam nesse sentido. Como a observação dos aglomerados revela que há presença efetiva de movimento relativamente superior nesses locais, talvez seja interessante, em estudos futuros, confrontar esse resultado com as expectativas dos consumidores em relação às aglomerações. Um percentual comparativamente elevado $(97,8 \%)$ de consumidores afirmou não ser difícil estacionar o carro nas aglomerações varejistas. Tal resultado pode decorrer da existência de estacionamentos (pagos) nessas áreas ou do fato de essa característica não ter despertado a atenção dos consumidores, que podem preferir o uso de transporte público. Ir aos clusters comerciais sem carro e ter de carregar as compras não foi considerado 'ruim' por $94,2 \%$ dos respondentes. Pode-se especular que as vantagens eventualmente encontradas nesses locais compensem o incômodo.

Deve-se reconhecer que $89,8 \%$ dos entrevistados afirmaram que, nos clusters comerciais, os produtos são todos de um mesmo fabricante ou fornecedor. Essa falta de opção pode contribuir para afastar os clientes, de acordo com o que apontou a literatura e os especialistas consultados. Do total de entrevistados (137 indivíduos), de acordo com o critério apresentado na seção do método empregado, 88 foram considerados clientes de clusters comerciais $(64,2 \%)$ e 49 não foram enquadrados como clientes de clusters comerciais $(35,8 \%)$. A amostra foi composta por 60 homens $(43,5 \%)$ e 77 mulheres $(55,8 \%)$. 


\subsection{Análise discriminante}

Objetivando a construção de uma abordagem orientada para a compreensão das variáveis de pesquisa associadas a um comportamento de compra preferencialmente vinculado a clusters comerciais, optou-se pela condução de uma análise discriminante. Utilizando-se o software IBM SPSS v.21.0, admitiu-se a variável dependente (dicotômica e nominal) como a condição de cliente de clusters comerciais do entrevistado (sim ou não). A análise discriminante múltipla, segundo Hair Jr. et al. (2009, p. 208), "encontra ampla aplicação em situações nas quais o objetivo principal é a determinação do grupo ao qual um objeto (por exemplo, uma pessoa, uma firma ou um produto) pertence". Como a amostra era de 137 entrevistados, foram utilizadas 24 variáveis (Quadro 5), respeitando-se a recomendação de natureza operacional proposta por Hair Jr. et al. (2009, p. 236) de ao menos cinco observações por variável independente. Optou-se pelo procedimento de entrada com todas as variáveis independentes simultaneamente, admitindo-se a probabilidade inicial de um objeto pertencer a cada grupo como 50\%. O modelo, quando aplicado aos dados de origem para a geração da função discriminante, resultou em 79,5\% de classificação correta, conforme Tabela 3, sugerindo uma capacidade preditiva importante. Entretanto, deve-se reconhecer que o valor da indicação de casos classificados adequadamente não raro é entendido na literatura de administração como relevante ou, ao menos, considerável (GONÇALVES; DIAS; MUNIZ, 2008; PIZZOL, 2004).

Tabela 4 - Resultado da classificação ${ }^{\mathrm{a}}$

\begin{tabular}{|c|c|c|c|c|c|}
\hline \multirow{2}{*}{\multicolumn{3}{|c|}{ Cliente de cluster? }} & \multicolumn{2}{|c|}{ Pertencimento previsto ao grupo } & \multirow{2}{*}{$\begin{array}{l}\text { Total } \\
\text { Não }\end{array}$} \\
\hline & & & Não & Sim & \\
\hline \multirow{4}{*}{ Original } & \multirow{2}{*}{ Contagem } & Não & 34 & 15 & 49 \\
\hline & & Sim & 18 & 70 & 88 \\
\hline & \multirow{2}{*}{$\%$} & Não & 69,4 & 30,6 & 100 \\
\hline & & Sim & 20,5 & 79,5 & 100 \\
\hline
\end{tabular}

a. Porcentagem de casos grupados classificados corretamente: 79,5\%.

Fonte: Autores.

O valor da função no centroide (função discriminante canônica não padronizada avaliada na média) do grupo do "sim" (clientes de clusters) é -0,402, e no grupo "não" (não clientes de clusters) é 0, 721. Foram obtidas duas funções discriminantes: (1) para elementos que são potenciais compradores de clusters (grupo "sim") e (2) para aqueles que não são 
potenciais compradores de clusters (grupo "não"). A Tabela 5 apresenta os valores dos coeficientes.

Tabela 5 - Coeficientes da função de classificação ${ }^{\mathrm{b}}$

\begin{tabular}{|l|c|c|}
\hline \multirow{2}{*}{ VARIÁVEIS DE PESQUISA } & \multicolumn{2}{c|}{ Cliente de cluster? } \\
\cline { 2 - 3 } & Não & Sim \\
\hline Sexo & $-0,986$ & $-1,689$ \\
\hline Comprou em cluster & 26,129 & 27,599 \\
\hline Foi e não comprou & 6,1 & 5,391 \\
\hline Desconhece x Conhece & 1,869 & 1,811 \\
\hline Compras Lentas x Rápidas & 1,709 & 1,554 \\
\hline Preços Altos x Baixos & 2,467 & 2,411 \\
\hline Variedade Pequena x Grande & 3,058 & 2,948 \\
\hline Conhec. Vend. Pequeno x Grande & $-1,777$ & $-1,611$ \\
\hline Encontrar prod. Difícil x Fácil & $-0,945$ & $-1,028$ \\
\hline Informação prod. Difícil x Fácil & 3,174 & 2,944 \\
\hline Localização Ruim x Boa & 1,252 & 1,618 \\
\hline Acesso Difícil x Fácil & 2,265 & 2,101 \\
\hline Achar complem. Difícil x Fácil & 0,517 & 0,859 \\
\hline Muito movimento & 81,667 & 83,149 \\
\hline Compras lentas & 1,812 & 2,162 \\
\hline Produtos não finos & 7,327 & 6,836 \\
\hline Produtos mesmo fabricante & 0,827 & 1,322 \\
\hline Vendedores enrolam & $-0,002$ & $-0,402$ \\
\hline Difícil achar o que procura & 8,65 & 7,909 \\
\hline Preços iguais a outros & 9,24 & 9,021 \\
\hline Não há segurança & $-5,189$ & $-5,622$ \\
\hline Estacionar é difícil & 78,07 & 76,364 \\
\hline Ruim ir a pé e carregar compras & 37,344 & 38,94 \\
\hline (Constante) & $-159,971$ & $-160,924$ \\
\hline
\end{tabular}

b. Função discriminante linear de Fisher.

Fonte: Autores.

As variáveis estão ordenadas por tamanho absoluto da correlação em relação à função. Como os resultados são padronizados, podem ser comparados e ordenados. De acordo com os coeficientes da Tabela 6, as características dos clusters comerciais mais importantes para atrair os consumidores são, em ordem decrescente de importância: (1) localização ser considerada boa, (2) facilidade para encontrar complementos para a compra principal, (3) condição de dificuldade para os clientes se deslocarem ao cluster a pé e terem que carregar as compras, (4) conhecimento dos vendedores sobre os produtos, (5) fato de os consumidores já terem comprado em clusters, (6) opinião dos consumidores de que nos clusters a compra é 
demorada, (7) os preços serem iguais aos de outros locais, (8) o quão bons os preços são, da facilidade para se informar sobre os produtos, (9) facilidade para encontrar produtos, (10) situação derivada de os consumidores considerarem os clusters muito movimentados, (11) variedade dos produtos e (12) produtos serem todos do mesmo fabricante.

Tabela 6 - Matriz da estrutura ${ }^{\mathrm{c}}$

\begin{tabular}{|c|c|}
\hline \multirow{2}{*}{ VARIÁVEIS DE PESQUISA } & Função 1 \\
\hline & (atração/clusters) \\
\hline Localização Ruim x Boa & $-0,393$ \\
\hline Achar prod. compl. Difícil x Fácil & $-0,392$ \\
\hline Vendedores enganam & 0,309 \\
\hline Ruim ir a pé e carregar compras & $-0,259$ \\
\hline Conhec. Vend. Pequeno x Grande & $-0,227$ \\
\hline Difícil achar o que procura & 0,224 \\
\hline Compras Lentas x Rápidas & 0,213 \\
\hline Estacionar é difícil & 0,207 \\
\hline Comprou em cluster & $-0,192$ \\
\hline Produtos não finos & 0,186 \\
\hline Compras lentas & $-0,174$ \\
\hline Foi e não comprou & 0,163 \\
\hline Desconhece x Conhece & 0,125 \\
\hline Preços iguais a outros & $-0,116$ \\
\hline Preços Altos x Baixos & $-0,098$ \\
\hline Informação prod. Difícil x Fácil & $-0,096$ \\
\hline Sexo & 0,087 \\
\hline Encontrar prod. Difícil x Fácil & $-0,084$ \\
\hline Acesso Difícil x Fácil & 0,078 \\
\hline Muito movimento & $-0,067$ \\
\hline Não há segurança & 0,041 \\
\hline Variedade Pequena x Grande & $-0,004$ \\
\hline Produtos mesmo fabricante & $-0,001$ \\
\hline
\end{tabular}

c. Correlações agrupadas dentro dos grupos entre variáveis discriminantes e funções discriminantes canônicas padronizadas.

Fonte: Autores.

A Tabela 6 oferece indicações também sobre as variáveis que afastam os consumidores dos clusters varejistas que, em ordem decrescente de importância, podem ser arroladas: (1) opinião de que neles os vendedores enganam (“enrolam”) os consumidores, (2) opinião de que neles é difícil achar o produto desejado, (3) velocidade das compras, (4) dificuldade para estacionar, (5) produtos vendidos não serem finos, (6) consumidores já terem 
ido a clusters comerciais e não terem comprado nada, (7) desconhecimento dos consumidores sobre os clusters, (8) sexo do consumidor, (9) facilidade do acesso ao local e (10) opinião de que não há segurança nesses locais.

\section{Considerações finais}

O cálculo dos alfas de Crombach e os resultados dos testes de Kayser-Meyer-Olkin e de Bartlett indicaram que o instrumento de coleta de dados desenvolvido pelos pesquisadores apresentou um nível de confiabilidade e de validade aceitável, o que abre a possibilidade de sua utilização em estudos futuros em contextos semelhantes. Esse fato pode ser considerado uma contribuição metodológica para o estudo dos clusters varejistas.

Os resultados desta pesquisa apontam para aspectos a serem incorporados no planejamento e na administração de negócios por gestores de varejo, assim como fornecem indicações para uma perspectiva estratégica de compreensão da atratividade de clusters varejistas, oferecendo oportunidade para futuros estudos de aprofundamento dessa abordagem ou de análise comparada entre clusters concorrentes.

A investigação de características dos clusters comerciais responsáveis pela atração e afastamento de clientes e a avaliação da importância relativa dessas características nos potenciais de atração e de afastamento, conforme descrito na seção 6.3 , conduziram a resultados relevantes sob a perspectiva de estratégia e gestão. A partir desses resultados infere-se que, ao analisar a possibilidade de comprar em clusters varejistas, os consumidores são atraídos por características ligadas à conveniência e a sua experiência anterior em clusters, ao passo que a falta de conveniência e a percepção de que os produtos dos clusters não são finos afastam-nos dos clusters. Nota-se que, apesar de os consumidores abrirem mão do conforto e ou status associado ao ato de comprar em outras configurações varejistas (como os shopping centers), o preço dos produtos não é um fator decisivo para a decisão de comprar nos clusters. Observe-se que a palavra "preço" ocupa apenas a sétima e oitava posições na Tabela 6. A possibilidade de fixar preços semelhantes aos de outras formas de varejo que apresentam custos de operação mais elevados talvez seja uma evidência empírica de que a situação de cluster favorece a rentabilidade das empresas, conforme previsto por Kultti (2011). Vale lembrar que, em um estudo realizado na cidade de São Paulo, Siqueira et al. (2012) observaram que lojas em clusters apresentaram resultados financeiros superiores aos de lojas semelhantes, mas isoladas. 
A análise das condições de atração e afastamento de consumidores para clusters comerciais necessariamente deve considerar que os resultados decorrem da percepção de indivíduos sobre esses agrupamentos, em especial de sua avaliação da relação entre benefícios e esforços de acesso a essa configuração particular de oferta.

Nesse sentido, as características apontadas no estudo revelam antes uma associação entre posições de potenciais compradores e os principais atributos vinculados à imagem que desenvolveram de clusters comerciais. Portanto, posições aparentemente conflituosas (como "Incômodo do acesso a pé e transporte de compras" como fator de atração) devem ser entendidas enquanto uma associação de variável e comportamento de consumidor (pessoas que se incomodam com o acesso a pé e transporte de compras apresentam maior probabilidade de decisão por compra em clusters).

\section{Referências}

AAKER, D. A.; KUMAR, V.; DAY, G. S. Pesquisa de marketing. 2. ed. São Paulo: Atlas, 2004.

ALTENBURG, T.; MEYER-STAMER, J. How to promote Clusters: policy experiences from Latin America. World Development, v. 27, n. 9, p. 1963-1713, 1999.

ARENTZE, T. A.; OPPEWAL, H.; TIMMERMANS, H. J. P. A multipurpose shopping trip model to assess retail agglomeration effects. Journal of Marketing Research, v. 42, n. 1, p. 109-115, 2005.

ARIKAN, A. Interfirm knowledge exchanges and the knowledge creation capability of clusters. Academy of Management Review, v. 34, n. 4, p. 658-677, 2009.

BECATTINI, G. Italian industrial districts: problems and perspectives. International Studies of Management and Organisation, n. 21, p. 83-90, 1991.

BEISEL, J. L. Retailing. 2. ed. New York: McMillan Publishing, 1993.

BERMAN, B.; EVANS, J. R. Retail management: a strategic approach. Upper Saddle River, NJ: Prentice-Hall, 2001. 
BIBA, G.; THÉRIAULT, M.; VILLENEUVE, P. Y.; DES ROSIERS, F. Aires de marché et choix des destinations de consommation pour les achats réalisés au cours de la semaine: Le cas de la región de Québec. The Canadian Geographer/Le Géographe Canadien, v. 52, n. 1, p. 38-63, 2008.

BOARI, C.; ODORICI, V.; ZAMARIAN, M. Clusters and rivalry: does localization really matter?. Scandinavian Journal of Management, v. 19, n. 4, p. 467-489, 2003.

BRANDENBURGUER, A. M.; NALEBUFF, B. J. Co-Opetição. São Paulo: Rocco, 1999.

CHURCHILL, G. A. A paradigm for developing better measures of marketing constructs, Journal of Marketing Research, p. 64-78, 1979.

COOPER, D. R.; SCHINDLER, P. S. Métodos de pesquisa em administração. 7. ed. São Paulo: Bookman, 2003.

DONAIRE, D.; GASPAR, M. A.; SILVA, R. S.; FITTIPALDI, M. A. S. Competitividade de clusters comerciais: estudo sobre uma aglomeração de pequenas lojas de veículos no município de São Paulo. Revista da Micro e Pequena Empresa, Campo Limpo Paulista, v. 7, n. 3, p. 64-78, 2013.

EL-ADLY, M. I. Shopping malls attractiveness: a segmentation approach. International Journal of Retail \& Distribution Management, v. 35, n. 11, p. 936-950, 2007.

FERNANDES, A. C.; LIMA, J. P. R. Cluster de serviços: contribuições conceituais com base em evidências do polo médico do Recife. Nova Economia, v. 16, n. 1, p. 11-47, 2006.

GONÇALVES, C. A.; DIAS, A. T.; MUNIZ, R. M. Análise discriminante das relações entre fatores estratégicos, indústria e desempenho em organizações brasileiras atuantes na indústria manufatureira. Revista de Administração Contemporânea - RAC, Curitiba, v. 12, n. 2, p. 287-311, 2008.

GORDON, W.; LANGMAID, R. Contemporary qualitative market research. Hampshire: Gower Publishing Limited, 1988.

HAIR JR., J. F.; BLACK, W. C.; BABIN, B. J.; ANDERSON, R. E.; TATHAM, R. L. Análise multivariada de dados. 6. ed. Porto Alegre: Bookman, 2009. 
HORTON, F. E. Location factors as determinants of consumer attraction to retail firms. Annals of the Association of American Geographers, v. 58, n. 4, p. 787-801, 1968.

HOTELling, H. Stability in competition. The Economic Journal, v. 39, n. 153. p. 41-57, 1929.

INGENE, C. A. Productivity and functional shifting in spatial retailing: private and social perspectives. Journal of Retailing and Consumer Services, v. 60, n. 3, p. 15-26, 1984.

KIM, Y. K. Consumer value: an application to mall and internet shopping. International Journal of Retail and Distribution Management, v. 30, n. 11/12, p. 595-602, 2002.

KINNEAR, T. C.; TAYLOR, J. R. Marketing research: An Applied Approach. $5^{\text {th }}$ ed. Maidenhead: McGraw-Hill, 1996.

KULTTI, K. Sellers like clusters. B. E. Journal of Theoretical Economics: Contributions to Theoretical Economics, v. 11, n. 1, p. 1-26, 2011.

LEVY, M.; WEITZ, B. A. Administração de varejo. São Paulo: Atlas, 2000.

LEE-ROSS, D. An exploratory study of the contextual stability of SERVQUAL amongst three retail Clusters in far North Queensland. Journal of Place Management and Development, v. 1, n. 1, p. 46-61, 2008.

LITZ, R. A.; RAJAGURU, G. Does small store location matter? A test of three classic theories of retail location. Journal of Small Business and Entrepreneurship, v. 21, n. 4, p. 477-492, 2008.

MALHOTRA, N. K. Pesquisa de marketing. 4. ed. Porto Alegre: Artmed Bookman, 2006.

MALMBERG, A.; POWER, D. (How) Do (firms in) Clusters create knowledge?. Industry and Innovation, v. 12, n. 4, p. 409-431, 2005.

MARIETTO, M. L.; SANCHES, C. Estratégia com prática: um estudo das práticas da ação estratégica no cluster de lojas comerciais da rua das noivas em São Paulo. Revista Pensamento Contemporâneo em Administração, Rio de Janeiro, v. 7, n. 3, p. 38-58, 2013. 
MARSHALL, A. Princípios de economia. v. 1. São Paulo: Abril Cultural, 1982.

MARTIN, S; MAYER, H. Sustainability, clusters, and competitiveness: Introduction to Focus Section. Economic Development Quarterly, v. 22, n. 4, p. 272-276, 2008.

MASCENA, K. M. Cunha de; FIGUEIREDO, F. Cruz; BOAVENTURA, J. M. G. Clusters e APLs: análise bibliométrica das publicações nacionais no período de 2000 a 2011 . RAE Revista de Administração de Empresas, v. 53, n. 5, p. 454-468, 2013. Disponível em: <http://www.redalyc.org/articulo.oa?id=155128126004>.

MASON, J. B.; BURNS, D. J. Retailing. 6. ed. Houston: Dame Publications, 1998.

MCDANIEL, C.; GATES, R. Pesquisa de marketing. São Paulo: Pioneira Thomson Learning, 2003.

NELSON, R. L. The selection of retail locations. New York: Dodge, 1958.

NOVELLI, M.; SCHMITZ, B.; SPENCE, T. Networks, Clusters and innovation in tourism: A UK experience. Tourism Management, v. 27, n. 6, p. 1141-1152, 2006.

NUNNALlY, J. C.; BERNSTEIN, I. H. Psychometric theory. $3^{\text {rd }}$ ed. New York: McGrawHill, 1994.

PARENTE, J. Varejo no Brasil: gestão e estratégia. São Paulo: Atlas, 2000.

PERRY, M. Business Clusters: an international perspective. New York: Routledge, 2005.

PIORE, M. E.; SABEL, C. The second industrial divide: possibilities for prosperity. Nova York: Basic Books, 1984.

PIZZOL, S. J. S. Combinação de grupos focais e análise discriminante: um método para tipificação de sistemas de produção agropecuária. Revista de Economia e Sociologia Rural, Brasília, v. 42, n. 3, 2004.

PORTER, M. E. The Competitive Advantage of Nations. New York: The Free Press, 1990. 
p. 77-90, 1998.

. Clusters and the new economics of competition. Harvard Business Review,

PROUDFOOT, M. J. City Retail Structure. Economic Geography, v. 13, n. 4, p. 425-428, 1937.

ROGERS, A. A stochastic analysis of the spatial clustering of retail establishments. Journal of the American Statistical Association, v. 60, n. 312, p. 1094-1104, 1965.

ROSENFELD, S. A. Bringing business Clusters into the mainstream of economic development. European Planning Studies, v. 5, n. 1, p. 3-23, 1997.

RUSSELL, J. E. Using a retail location game to explore Hotelling's Principle of Minimum Differentiation. Business Education Innovation Journal. v. 5, n. 2, p. 48-52, 2013.

SIQUEIRA, J. P. L.; TELLES, R.; HOURNEAUX JR., F.; TARTARELI, R. Competitividade de lojas isoladas e em clusters: um estudo comparativo na cidade de São Paulo. Espacios, v. 33, n. 4, 2012.

SCHIMITZ, H. On the clustering of small firms. IDS Bulletin, v. 23, n. 3, 1992.

SCHMITZ, H.; NADVI, K. Clustering and industrialization: introduction. World Development, v. 27, n. 9, p. 1503-1514, 1999.

SZAFIR-GOLDSTEIN, C.; TOLEDO, G. L. Competição e cooperação em Clusters industriais: estágios e políticas. Anais do VII SEMEAD, 2004.

TERENCE, A. C. F.; ESCRIVÃO, F. E. Abordagem quantitativa, qualitativa e a utilização de pesquisa-ação. In: XXVI Encontro Nacional de Engenharia de Produção - ENEGEP, Fortaleza. Anais... Fortaleza: ABEPRO, 2006.

TELLER, C.; ELMS, J. Managing the attractiveness of evolved and created retail agglomerations formats. Marketing Intelligence \& Planning, v. 28, n. 1, p. 25-45, 2010.

Urban place marketing and retail agglomeration customers.

Journal of Marketing Management, v. 28, n. 5/6, p. 546-567, 2012. 
; THOMSON, J. A.; PADDISON, A. R. Place marketing and urban retail agglomerations: An examination of shoppers' place attractiveness perceptions. Place Branding and Public Diplomacy, v. 6, n. 2, p. 124-133, 2010.

; REUTTERER, T. The evolving concept of retail attractiveness: What makes retail agglomerations attractive when customers shop at them?. Journal of Retailing and Consumer Services, v. 15, n. 3, p. 127-143, 2008.

; SCHNEDLITZ, P. Hedonic and utilitarian shopper types in evolved and created retail agglomerations. The International Review of Retail, Distribution and Consumer Research, v. 18, p. 55-93, 2008.

TELLES, R.; ALTHEMAN, E.; SIQUEIRA, J. P. L.; ROMBOLI, S. M. Clusters comerciais: um estudo sobre concentrações de bares na cidade de São Paulo. Gestão \& Regionalidade, v. 27, n. 81, p. 32-45, 2011.

; SIQUEIRA, J. P. L.; DONAIRE, D.; GASPAR, M. A. Atratividade em clusters comerciais: um estudo comparativo de dois clusters comerciais da cidade de São Paulo. Gestão \& Regionalidade, São Caetano do Sul, v. 29, n. 85, p. 47-62, 2013.

VAN DER VELDE, M.; JANSEN, P.; ANDERSON, N. Guide to management research methods. Oxford: Blackwell Publishing Ltd., 2004.

YANG, Z.; HAO, P.; CAI, J. Economic clusters: A bridge between economic and spatial policies in the case of Beijing. Cities. v. 42, p. 171-185, 2015.

ZACCARELLI, S. B. Estratégia e sucesso nas empresas. São Paulo: Saraiva, 2004.

; TELlES, R.; SIQUEIRA, J. P. L.; BOAVENTURA, J. M. G.; DONAIRE, D. Clusters e redes de negócios: uma nova visão para a gestão dos negócios. São Paulo: Atlas, 2008. 